\title{
Fermions in a mixed vector-scalar double-step potential via continuous chiral transformation
}

\author{
W.M. Castilho a and A.S. de Castro ${ }^{\mathrm{b}}$ \\ UNESP - Campus de Guaratinguetá, Departamento de Física e Química, 12516-410 Guaratinguetá SP, Brazil
}

Received: 12 November 2015 / Revised: 6 January 2016

Published online: 13 April 2016 - (c) Società Italiana di Fisica / Springer-Verlag 2016

\begin{abstract}
The behaviour of fermions in the background of a double-step potential is analyzed with a general mixing of scalar and vector couplings via continuous chiral-conjugation transformation. Provided the vector coupling does not exceed the scalar coupling, a Sturm-Liouville approach for the double-step potential shows that the transmission coefficient exhibits oscillations and that a finite set of intrinsically relativistic bound-state solutions might appear as poles of the transmission amplitude in a strong coupling regime. An isolated bound-state solution resulting from coupled first-order equations might also come into sight. It is also shown that all those possible bound solutions disappear asymptotically as one approaches the conditions for the realization of the so-called spin and pseudospin symmetries in a four-dimensional space-time. Furthermore, we show that due to the additional mass acquired by the fermion from the scalar background the high localization of the fermion in an extreme relativistic regime does not violate the Heisenberg uncertainty principle.
\end{abstract}

\section{Introduction}

The Dirac Hamiltonian with a mixing of scalar potential and time component of vector potential in a four-dimensional space-time is invariant under an $S U(2)$ algebra when the difference between the potentials, or their sum, is a constant [1]. The near realization of these symmetries may explain degeneracies in some heavy meson spectra (spin symmetry) [2,3] or in single-particle energy levels in nuclei (pseudospin symmetry) [3-38]. When these symmetries are realized, the energy spectrum does not depend on the spinorial structure, being identical to the spectrum of a spinless particle [39]. Despite the absence of spin effects in $1+1$ dimensions, many attributes of the spin and pseudospin symmetries in four dimensions are preserved.

In a pioneering work, Jackiw and Rebbi [40] have shown that massless fermions coupled to scalar fields with kink-like profiles in $1+1$ dimensions develops quantum states with fractional fermion number due to the zero-mode solution. This phenomenon has been seen in certain polymers such as polyacetylene [41]. Later, charge fractionization into irrational numbers was shown to emerge in a model without charge-conjugation symmetry [42]. Charge values with irrational numbers are present in a certain discrete model of diatomic polymers [43] which is related to chargeconjugation-invariance violation in the continuum limit [44], realizing the charge fractionization envisioned in ref. [42].

Recently the complete set of solutions for the kink-like scalar potential behaving like $\tanh x / \lambda$ has been considered for massless fermions in ref. [45], and for massive fermions in ref. [46]. Kink-like profiles violating the charge conjugation symmetry by addition of a time component of a vector potential has also been considered in the literature. The complete set of solutions for massive fermions under the influence of a kink-like scalar potential added by the time component of a vector potential with the same functional form was considered in refs. [47] and [48], in ref. [47] for the background field behaving like $\operatorname{sgn} x$, and in ref. [48] for the background behaving like $\tanh x / \lambda$. In refs. [47] and [48], it has been shown that the Dirac equation with a scalar potential plus a time component of vector potential of the same functional form is manageable if the vector coupling does not exceed the scalar coupling, and that the bound states for mixed scalar-vector potentials with the kink-like profiles $\operatorname{sgn} x$ and $\tanh x / \lambda$ are intrinsically relativistic solutions. Furthermore, it has been shown that the fermion can be confined in a highly localized region of space under a very strong field without any chance for spontaneous pair production related to Klein's paradox. In a more recent work [49],

\footnotetext{
a e-mail: castilho.w@gmail.com

b e-mail: castro@pq.cnpq.br
} 
it has been shown that the existence of such intrinsically relativistic bound states is ensured for any mixed scalar-vector potential with a kink-like profile.

Elsewhere, it has been shown that the double-step potential can furnish intrinsically relativistic bound states when one considers a pseudoscalar coupling in the Dirac equation [50] or a nonminimal vector coupling in the Duffin-KemmerPetiau equation for spinless particles [51] and spin-1 particles [52]. However, the double-step potential does not seem to have received any attention regarding scalar and vector couplings in the Dirac equation. In what follows the mixing formalism developed in $[47,48]$ is addressed to the double-step potential. We shall present a fairly complete account of the problem and show that, in contrast to the case of a sign step potential of ref. [47] and alike the smooth step potential of ref. [48], aside from the isolated solution the spectrum might consist of a finite set of bound-state solutions under a strong-coupling regime. Furthermore, we shall show that the fermion can be trapped in a highly localized region under an extreme relativistic regime and that this high localization maintains the single-particle interpretation of the Dirac theory because the fermion acquires an additional mass coming from its interaction with the scalar-field background. A distinctive aspect of this problem is that the transmission coefficient exhibits oscillations. The limit where the double-step potential becomes the sign potential is also considered. We begin by reviewing those results of refs. [47,48] and [49] which are directly relevant for the present work.

\section{Mixed scalar-vector interactions}

Consider the Lagrangian density for a massive fermion

$$
L=\bar{\Psi}\left(i \hbar c \gamma^{\mu} \partial_{\mu}-I_{m} c^{2}-V\right) \Psi,
$$

where $\hbar$ is the constant of Planck, $c$ is the velocity of light, $I$ is the unit matrix, $m$ is the mass of the free fermion and the square matrices $\gamma^{\mu}$ satisfy the algebra $\left\{\gamma^{\mu}, \gamma^{\nu}\right\}=2 I g^{\mu \nu}$. The spinor adjoint to $\Psi$ is defined by $\bar{\Psi}=\Psi^{\dagger} \gamma^{0}$. For vector and scalar interactions the matrix potential is written as $V=\gamma^{\mu} A_{\mu}+I V_{s}$. Requiring $\left(\gamma^{\mu}\right)^{\dagger}=\gamma^{0} \gamma^{\mu} \gamma^{0}$, one finds the continuity equation $\partial_{\mu} J^{\mu}=0$, where the conserved current is $J^{\mu}=c \bar{\Psi} \gamma^{\mu} \Psi$. Equation (1) leads to the Hamiltonian form for the Dirac equation $i \hbar \partial \Psi / \partial t=H \Psi$. In $1+1$ dimensions $\Psi$ is a $2 \times 1$ matrix, the metric tensor is $g^{\mu \nu}=\operatorname{diag}(1,-1)$ and the Hamiltonian is given by

$$
H=\gamma^{5} c\left(p_{1}+\frac{A_{1}}{c}\right)+I A_{0}+\gamma^{0}\left(m c^{2}+V_{s}\right),
$$

where $\gamma^{5}=\gamma^{0} \gamma^{1}$. Assuming time-independent potentials, one can write $\Psi(x, t)=\psi(x) \exp (-i E t / \hbar)$ in such a way that the time-independent Dirac equation becomes $H \psi=E \psi$. Meanwhile $J^{\mu}=c \bar{\psi} \gamma^{\mu} \psi$ is time independent and $J^{1}$ is uniform. From now on, we make $A_{1}=0$ and use an explicit representation for the $2 \times 2$ matrices $\gamma$ as $\gamma^{0}=\sigma_{3}$ and $\gamma^{1}=i \sigma_{2}$. Here, $\sigma_{2}$ and $\sigma_{3}$ stand for the Pauli matrices.

The charge-conjugation operation is accomplished by the transformation $\psi \rightarrow \sigma_{1} \psi^{*}$ followed by $A_{0} \rightarrow-A_{0}$, $V_{s} \rightarrow V_{s}$ and $E \rightarrow-E$ [53]. The chiral-conjugation operation $\psi \rightarrow \gamma^{5} \psi$ (according to ref. [54]) is followed by the changes of the signs of $V_{s}$ and $m$, but not of $A_{0}$ and $E$ [53]. One sees that the charge-conjugation and the chiralconjugation operations interchange the roles of the upper and lower components of the Dirac spinor. The continuous chiral transformation (see, e.g., [55]) is induced by the unitary operator

$$
U(\theta)=\exp \left(-\frac{\theta}{2} i \gamma^{5}\right)
$$

where $\theta$ is a real quantity such that $0 \leq \theta \leq \pi$. It allows one to write

$$
h \phi=E \phi, \quad \phi=U \psi, \quad h=U H U^{-1}
$$

with

$$
h=\sigma_{1} c p_{1}+I A_{0}+\sigma_{3}\left(m c^{2}+V_{s}\right) \cos \theta-\sigma_{2}\left(m c^{2}+V_{s}\right) \sin \theta,
$$

with $A_{0}$ and $V_{s}$ related by

$$
A_{0}=V_{s} \cos \theta
$$

and eliminating $A_{0}$ in favor of $V_{s}$, one can rewrite the Dirac equation in terms of the upper $\left(\phi_{+}\right)$and lower $\left(\phi_{-}\right)$ components of $\phi$ as

$$
\begin{aligned}
& \hbar c \frac{\mathrm{d} \phi_{+}}{\mathrm{d} x}+\left(m c^{2}+V_{s}\right) \sin \theta \phi_{+}=i\left(E+m c^{2} \cos \theta\right) \phi_{-} \\
& \hbar c \frac{\mathrm{d} \phi_{-}}{\mathrm{d} x}-\left(m c^{2}+V_{s}\right) \sin \theta \phi_{-}=i\left[E-\left(m c^{2}+2 V_{s}\right) \cos \theta\right] \phi_{+} .
\end{aligned}
$$


For $E \neq-m c^{2} \cos \theta$, one finds

$$
J^{1}=\frac{2 \hbar c^{2}}{E+m c^{2} \cos \theta} \operatorname{Im}\left(\phi_{+}^{*} \frac{\mathrm{d} \phi_{+}}{\mathrm{d} x}\right)
$$

Furthermore,

$$
-\frac{\hbar^{2}}{2} \frac{\mathrm{d}^{2} \phi_{+}}{\mathrm{d} x^{2}}+V_{\text {eff }} \phi_{+}=E_{\text {eff }} \phi_{+}
$$

with

$$
V_{\text {eff }}=\frac{\sin ^{2} \theta}{2 c^{2}} V_{s}^{2}+\frac{m c^{2}+E \cos \theta}{c^{2}} V_{s}-\frac{\hbar \sin \theta}{2 c} \frac{\mathrm{d} V_{s}}{\mathrm{~d} x}
$$

and

$$
E_{\text {eff }}=\frac{E^{2}-m^{2} c^{4}}{2 c^{2}} .
$$

In this way one can solve the Dirac problem for determining the possible discrete or continuous eigenvalues of the system by referring to the solution of the related Schrödinger problem because $\phi_{+}$is a square-integrable function.

Defining

$$
v(x)=\int^{x} \mathrm{~d} y V_{s}(y)
$$

the solutions for $(7 \mathrm{a})$ and $(7 \mathrm{~b})$ with $E=-m c^{2} \cos \theta$ are

$$
\begin{aligned}
& \phi_{+}=N_{+}, \\
& \phi_{-}=N_{-}-2 \frac{i}{\hbar c} N_{+}\left[m c^{2} x+v(x)\right] \cos \theta,
\end{aligned}
$$

for $\sin \theta=0$, and

$$
\begin{aligned}
& \phi_{+}=N_{+} \exp \left\{-\frac{\sin \theta}{\hbar c}\left[m c^{2} x+v(x)\right]\right\} \\
& \phi_{-}=N_{-} \exp \left\{+\frac{\sin \theta}{\hbar c}\left[m c^{2} x+v(x)\right]\right\}+i \phi_{+} \cot \theta
\end{aligned}
$$

for $\sin \theta \neq 0 . N_{+}$and $N_{-}$are normalization constants. Note that these solutions isolated from the Sturm-Liouville problem can not describe scattering states and $J^{1}=2 c \operatorname{Re}\left(N_{+}^{*} N_{-}\right)$. A bound-state solution demands $N_{+}=0$ or $N_{-}=0$, because $\phi_{+}$and $\phi_{-}$are square-integrable functions. There is no bound-state solution for $\sin \theta=0$, and for $\sin \theta \neq 0$ the existence of a bound state solution depends on the asymptotic behaviour of $v(x)[56,57]$.

\section{Kink potentials}

Now we consider a kink-like potential with the asymptotic behaviour $V_{s}(x) \rightarrow \pm v_{0}$ as $x \rightarrow \pm \infty$, with $v_{0}=$ constant.

We turn our attention to scattering states for fermions with $E \neq-m c^{2} \cos \theta$ coming from the left. Then, $\phi$ for $x \rightarrow-\infty$ describes an incident wave moving to the right and a reflected wave moving to the left, and $\phi$ for $x \rightarrow+\infty$ describes a transmitted wave moving to the right or an evanescent wave. The upper component for scattering states is written as

$$
\phi_{+}=\left\{\begin{array}{cc}
A_{+} e^{+i k_{-} x}+A_{-} e^{-i k_{-} x}, & \text { for } \quad x \rightarrow-\infty \\
B_{ \pm} e^{ \pm i k_{+} x}, & \text { for } \quad x \rightarrow+\infty
\end{array}\right.
$$

where

$$
\hbar c k_{ \pm}=\sqrt{\left(E \mp v_{0} \cos \theta\right)^{2}-\left(m c^{2} \pm v_{0}\right)^{2}} .
$$

Therefore,

$$
J_{\gtrless}^{1}(-\infty)=\frac{2 \hbar c^{2} k_{-}}{E+m c^{2} \cos \theta}\left(\left|A_{ \pm}\right|^{2}-\left|A_{\mp}\right|^{2}\right), \quad \text { for } \quad E \gtrless-m c^{2} \cos \theta
$$

and

$$
J_{\gtrless}^{1}(+\infty)= \pm \frac{2 \hbar c^{2} \operatorname{Re} k_{+}}{E+m c^{2} \cos \theta}\left|B_{ \pm}\right|^{2}, \quad \text { for } \quad E \gtrless-m c^{2} \cos \theta
$$


If $E>-m c^{2} \cos \theta$, then $A_{+} e^{+i k_{-} x}\left(A_{-} e^{-i k_{-} x}\right)$ will describe the incident (reflected) wave, and $B_{-}=0$. On the other hand, if $E<-m c^{2} \cos \theta$, then $A_{-} e^{-i k_{-} x}\left(A_{+} e^{+i k_{-} x}\right)$ will describe the incident (reflected) wave, and $B_{+}=0$. Therefore, the reflection and transmission amplitudes are given by

$$
r_{\gtrless}=\frac{A_{\mp}}{A_{ \pm}}, \quad t_{\gtrless}=\frac{B_{ \pm}}{A_{ \pm}}, \quad \text { for } \quad E \gtrless-m c^{2} \cos \theta .
$$

The $x$-independent space component of the current allows us to define the reflection and transmission coefficients as

$$
R_{\gtrless}=\frac{\left|A_{\mp}\right|^{2}}{\left|A_{ \pm}\right|^{2}}, \quad T_{\gtrless}=\frac{\operatorname{Re} k_{+}}{k_{-}} \frac{\left|B_{ \pm}\right|^{2}}{\left|A_{ \pm}\right|^{2}}, \quad \text { for } \quad E \gtrless-m c^{2} \cos \theta .
$$

As for $E=-m c^{2} \cos \theta$, the existence of a bound-state solution requires $\left|v_{0}\right|>m c^{2}$ so that, $\operatorname{defining} \tilde{\phi}^{\mathrm{T}}=(1 i \cot \theta)$ for $v_{0}>+m c^{2}$, and $\tilde{\phi}^{\mathrm{T}}=\left(\begin{array}{ll}0 & 1\end{array}\right)$ for $v_{0}<-m c^{2}$, the eigenspinor behaves asymptotically as $\phi \sim \tilde{\phi} f$ with

$$
f=\exp \left\{-\frac{\sin \theta}{\hbar c}\left[\left|v_{0}\right|+m c^{2} \operatorname{sgn}\left(v_{0} x\right)\right]|x|\right\} .
$$

\section{The double-step potential}

Now we assume a scalar potential in the form

$$
V_{s}=v_{0}[\Theta(x-a)-\Theta(-x-a)]
$$

with $v_{0}$ and $a$ defined to be real numbers $(a>0)$ and $\Theta(x)$ is the Heaviside step function. Notice that $V_{s}$ is invariant under a coincidental change of the signs of $v_{0}$ and $x$, and that as $a \rightarrow 0$ the double-step approximates the sign potential already considered in ref. [47].

Our problem is to solve the set of eqs. (7a)-(7b) for $\phi$ and to determine the allowed energies for both classes of solutions sketched in sect. 2 .

\subsection{The case $\mathrm{E} \neq-\mathrm{mc}^{2} \cos \theta$}

For our model,

$$
V_{\text {eff }}=\left(V_{1}+V_{2}\right) \Theta(x-a)+\left(V_{1}-V_{2}\right) \Theta(-x-a)-\hbar \operatorname{sgn}\left(v_{0}\right) \sqrt{\frac{V_{1}}{2}}[\delta(x-a)+\delta(x+a)],
$$

where $\delta(x)=\mathrm{d} \Theta(x) / \mathrm{d} x$ is the Dirac delta function and the following abbreviations have been used:

$$
\begin{aligned}
V_{1} & =\frac{v_{0}^{2} \sin ^{2} \theta}{2 c^{2}}, \\
V_{2} & =v_{0} \frac{E \cos \theta+m c^{2}}{c^{2}} .
\end{aligned}
$$

For $V_{1}=0, V_{\text {eff }}$ is an ascendant (a descendant) double step if $V_{2}>0\left(V_{2}<0\right)$. For $V_{1} \neq 0$, the "effective potential" includes attractive (repulsive) delta functions at $x= \pm a$ if $v_{0}>0\left(v_{0}<0\right)$. It has plateaus given by $V_{1} \pm V_{2}$ for $x \gtrless \pm a$ $\left(V_{\text {eff }}=0\right.$ for $\left.|x|<a\right)$ and so we can consider scattering states for fermions coming from the left with $E_{\text {eff }}>V_{1}-V_{2}$. Due to the discontinuities (for $V_{1}=0$ ) or singularities (for $V_{1} \neq 0$ ) of $V_{\text {eff }}$ at $x= \pm a$ one should expect resonant transmission for certain values of $E_{\text {eff }}$. Furthermore, for $V_{2}>V_{1}$ and $E_{\text {eff }}<0$ one should expect nonprogressive waves in the region $|x|<a$ and no transmission whereas the transmission is ubiquitous for $V_{2} \leq 0$. Bound-state solutions for $\left|V_{2}\right|<V_{1}$ with $0<E_{\text {eff }}<V_{1}-\left|V_{2}\right|$ should be expected even if $v_{0}<0$ (delta functions apart, $\phi_{+}$is insensitive to simultaneous changes in the signs of $v_{0}$ and $\left.x\right)$. It is instructive to note that $V_{1}$ tends to vanish as $\left|v_{0}\right| / m c^{2} \rightarrow 0$ so that the effective potential becomes the double-step potential in a nonrelativistic scheme.

We demand that $\phi_{+}$be continuous at $x= \pm a$, that is

$$
\lim _{\varepsilon \rightarrow 0}\left(\left.\phi_{+}\right|_{x= \pm a+\varepsilon}-\left.\phi_{+}\right|_{x= \pm a-\varepsilon}\right)=0 .
$$

Otherwise, the differential equation for $\phi_{+}$would contain derivative of $\delta$-functions. Effects on $\mathrm{d} \phi_{+} / \mathrm{d} x$ in the neighbourhood of $x= \pm a$ can be evaluated by integrating the differential equation for $\phi_{+}$from $\pm a-\varepsilon$ to $\pm a+\varepsilon$ and taking the limit $\varepsilon \rightarrow 0$. The connection formulas for $\mathrm{d} \phi_{+} / \mathrm{d} x$ can be summarized as

$$
\lim _{\varepsilon \rightarrow 0}\left(\left.\frac{\mathrm{d} \phi_{+}}{\mathrm{d} x}\right|_{x= \pm a+\varepsilon}-\left.\frac{\mathrm{d} \phi_{+}}{\mathrm{d} x}\right|_{x= \pm a-\varepsilon}\right)=-\left.\frac{v_{0} \sin \theta}{\hbar c} \phi_{+}\right|_{x= \pm a} .
$$




\subsubsection{Scattering states}

We turn our attention to scattering states for fermions coming from the left as sketched in sect. 3. The upper component for scattering states on the entire space is written as

$$
\begin{aligned}
\phi_{+}= & {[1-\Theta(x+a)]\left(A_{+} e^{+i \zeta_{-} x / a}+A_{-} e^{-i \zeta_{-} x / a}\right) } \\
& +[\Theta(x+a)-\Theta(x-a)]\left(C_{+} e^{+i \xi x / a}+C_{-} e^{-i \xi x / a}\right) \\
& +\Theta(x-a) B_{ \pm} e^{ \pm i \zeta_{+} x / a}
\end{aligned}
$$

where

$$
\begin{aligned}
\zeta_{ \pm} & =a k_{ \pm}, \\
\xi & =\frac{a}{\hbar c} \sqrt{E^{2}-m^{2} c^{4}} .
\end{aligned}
$$

It is instructive to note that these quantities satisfy the constraint

$$
\zeta_{-}^{2}+\zeta_{+}^{2}=2\left(\xi^{2}-v^{2}\right)
$$

where

$$
v=\frac{a v_{0} \sin \theta}{\hbar c}
$$

with $\phi_{+}$given by (27) and for $E>-m c^{2} \cos \theta$, conditions (25) and (26) imply into the relative amplitudes

$$
\begin{aligned}
\frac{A_{-}}{A_{+}} & =e^{-2 i \zeta_{-}} \frac{\left(\zeta_{-}-\zeta_{+}\right) \mu+i \sigma_{-}}{\left(\zeta_{-}+\zeta_{+}\right) \mu-i \sigma_{+}}, \\
\frac{B_{+}}{A_{+}} & =e^{-i\left(\zeta_{-}+\zeta_{+}\right)} \zeta_{-} \frac{2 \xi}{\left(\zeta_{-}+\zeta_{+}\right) \mu-i \sigma_{+}} \\
\frac{C_{ \pm}}{A_{+}} & =e^{-i \zeta_{-}} \zeta_{-} \frac{\eta_{ \pm}}{\left(\zeta_{-}+\zeta_{+}\right) \mu-i \sigma_{+}}
\end{aligned}
$$

where we have set

$$
\begin{aligned}
\mu & =\xi \cos 2 \xi-v \sin 2 \xi \\
\sigma_{ \pm} & =2 \xi v \cos 2 \xi+\frac{\left(\zeta_{-} \pm \zeta_{+}\right)^{2}}{2} \sin 2 \xi \\
\eta_{ \pm} & =\left(\xi \pm \zeta_{+}\right) \cos \xi-v \sin \xi \mp i\left[v \cos \xi+\left(\xi \pm \zeta_{+}\right) \sin \xi\right] .
\end{aligned}
$$

For $E<-m c^{2} \cos \theta$, the amplitudes can be obtained by taking the complex conjugate of the right-hand side of (31) and exchanging the signs of the subscripts of the amplitudes. It follows that

$$
T=T_{\gtrless}=\frac{32 \xi^{2} \zeta_{-} \operatorname{Re} \zeta_{+}}{c_{1} \cos 4 \xi+c_{2}},
$$

where

$$
\begin{aligned}
& c_{1}=\left(\zeta_{-}^{2}-\zeta_{+}^{2}\right)^{2}-16 \xi^{2} v^{2}, \\
& c_{2}=8 \xi^{2}\left(\zeta_{-}+\zeta_{+}\right)^{2}-c_{1},
\end{aligned}
$$

not considering whether $E>-m c^{2} \cos \theta$ or $E<-m c^{2} \cos \theta$. Because we chose fermions coming from the left, the transmission coefficient is not invariant under the change of $v_{0}$ by $-v_{0}$, however, this symmetry is exact when $T \neq 0$. The transmission coefficient does not depends on the sign of $E$ in the case of a pure scalar coupling. It is true that $\xi$ imaginary makes $\zeta_{-}$imaginary. Naturally, the transmission does not exist neither does the incidence if $\xi$ is small enough to make $\zeta_{-}$imaginary. The transmission is possible only if $\zeta_{-}$and $\zeta_{+}$are real quantities and this fact imposes a cutoff on $\xi$. As a function of $\xi$, the transmission coefficient rises from zero for a certain $\xi$, oscillates between extreme 


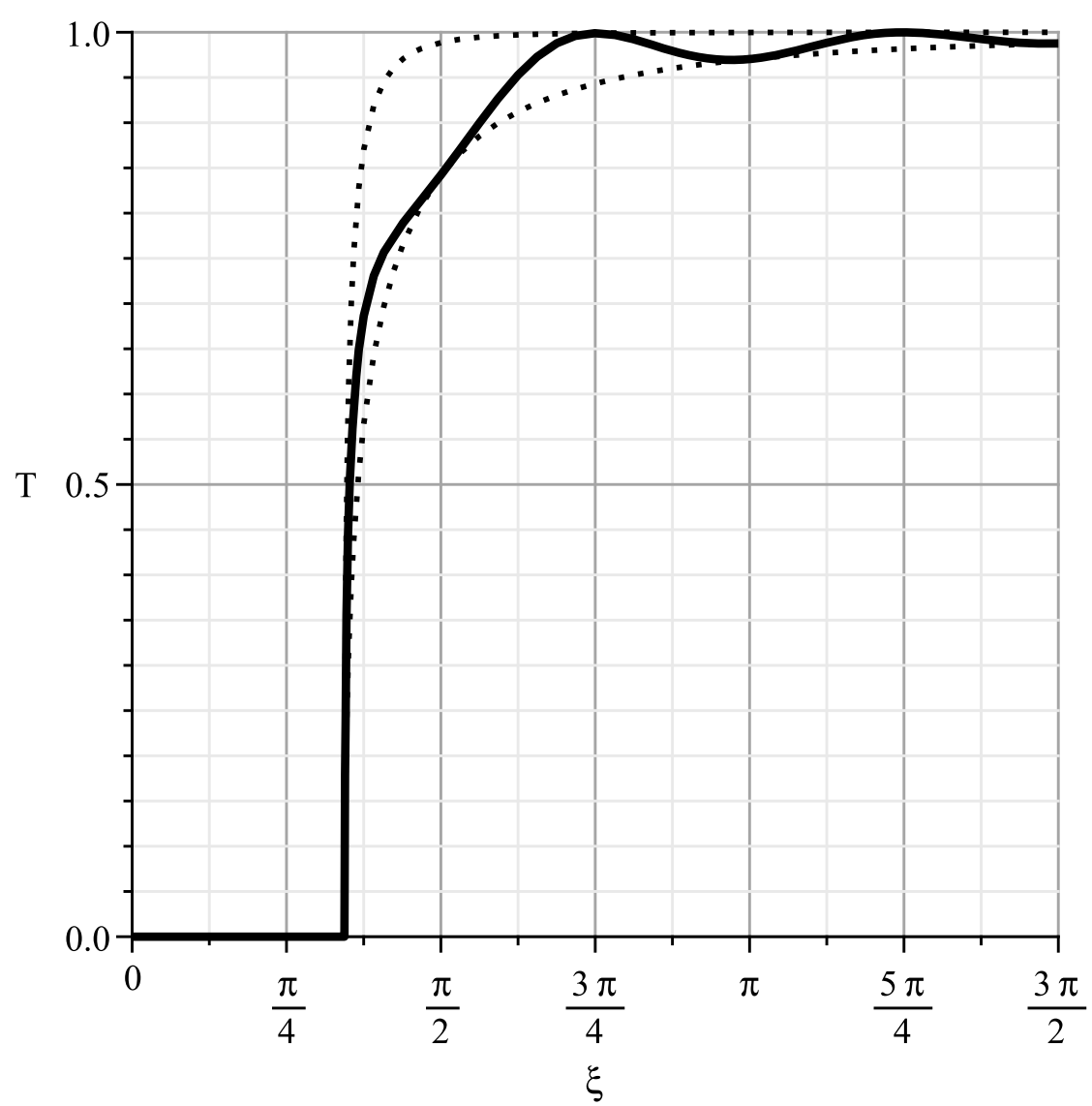

Fig. 1. Transmission coefficient as a function of $\xi$ (continuous line) for $v_{0} / m c^{2}=1, a / \lambda_{C}=1 / 2$ and $\theta=3 \pi / 8$. The dotted lines osculate the minima and maxima of the transmission coefficient.

values and approaches $T=1$ as $\xi \rightarrow \infty$. This is in contrast to the transmission coefficient for the sign potential [47], where no oscillation exists. The maxima of $T$ occur when

$$
\xi_{n}=\left(n-\frac{1}{2}\right) \frac{\pi}{2}, \quad n_{\min }<n=1,2,3, \ldots
$$

and they are osculated by the function

$$
T_{\max }=\frac{4 \xi^{2}}{\left(\zeta_{-}+\zeta_{+}\right)^{2}} \frac{4 \zeta_{-} \operatorname{Re} \zeta_{+}}{\left(\zeta_{-}+\zeta_{+}\right)^{2}+(2 v)^{2}},
$$

$n_{\text {min }}$ is associated with the cutoff on $\xi$. On the other hand, the minima of $T$ occur when $\xi=(n+1) \pi / 2$, and they are osculated by the function

$$
T_{\min }=\frac{4 \zeta_{-} \operatorname{Re} \zeta_{+}}{\left(\zeta_{-}+\zeta_{+}\right)^{2}+(2 v)^{2}} .
$$

The maxima (or minima) are regularly separated from each other by $\xi=\pi / 2$ and seen as a function of $E$ they are more scarce the small $a$. As a matter of fact, as $a \rightarrow 0$ one finds the transmission coefficient for the sign step potential [47]: $T \simeq T_{\min }$. It is also worthwhile to note that the oscillations in the transmission coefficient only manifest in a nonrelativistic scheme if $a \gg \lambda_{C}$. Figure 1 shows the transmission coefficient as a function of $\xi$ for $v_{0} / m c^{2}=1$, $a / \lambda_{C}=1 / 2$ and $\theta=3 \pi / 8$.

\subsection{Bound states}

Following the previous qualitative considerations, we discuss the existence of bound states. One way to identify the possible bound-state solutions is to look for the poles of the transmission amplitude in the complex $\zeta$-plane. If one 
considers the transmission amplitude $t_{ \pm}$in (19) as a function of the complex variables $\zeta_{ \pm}$one sees that bound states would be obtained by the poles along the imaginary axis of the complex $\zeta$-plane. These poles require $A_{ \pm}=0$ and $B_{ \pm} \neq 0$, corresponding to $E \gtrless-m c^{2} \cos \theta$. Furthermore, square-integrability of $\phi_{+}$demands $\zeta_{-}= \pm i\left|\zeta_{-}\right|$and $\zeta_{+}= \pm i\left|\zeta_{+}\right|$. Therefore, the bound states would occur for $\Sigma=\sigma_{+} / \mu$, even if $\xi=0$. Here $\Sigma=\left|\zeta_{-}\right|+\left|\zeta_{+}\right|$. Hence,

$$
\Sigma=2 v
$$

or

$$
\Sigma=-2 \xi \cot 2 \xi
$$

Equations (38) and (39) would determinate the energies of the bound states. Notice that any possible solution demands $\Sigma>0$.

It is easy to see that (38) is impossible for $v<0$. Equation (38) has not even solution for $v>0$. In fact, squaring (38) results in the form of a second-degree algebraic equation

$$
E^{2}+2 m c^{2} E \cos \theta+m^{2} c^{4} \cos ^{2} \theta=0
$$

which presents just one solution for $v>0$, viz. $E=-m c^{2} \cos \theta$, without regard to $a$. Evidently, it is not a proper solution of the problem.

The remaining quantization condition, eq. (39), has no solution if $\xi$ is imaginary because its right-hand side would be a negative number. For $\xi \in \mathbb{R}\left(|E|>m c^{2}\right)$, though, it dictates that the spectrum depends on the mixing angle and that it is symmetrical about $E=0$ in the case of a pure scalar coupling. The sign of $v_{0}$ does not effect the spectrum but $a$ does. In the case of a massless fermion the spectrum does not change when the mixing angle changes from $\pi / 2-\varepsilon$ to $\pi / 2+\varepsilon$. From $(28)$ one sees that $\zeta_{-}$and $\zeta_{+}$are imaginary numbers only when $\left|v_{0}\right|>m c^{2}$ so that those solutions only survive in a relativistic regime. Equation (39) also dictates that $\xi \cot 2 \xi<0$ so that $\xi>\pi / 4$. In addition, eq. (29) becomes $\left|\zeta_{-}\right|^{2}+\left|\zeta_{+}\right|^{2}=2\left(v^{2}-\xi^{2}\right)$ for bound states and so $\xi<|v|$. In this way we have to search for solutions in the interval $\pi / 4<\xi<|v|$, corresponding to

$$
\left(\frac{\pi \hbar c}{4 a}\right)^{2}<E^{2}-m^{2} c^{4}<\left(v_{0} \sin \theta\right)^{2}
$$

At once, we can state that the possible spectrum for bound states calls for $\sin \theta \neq 0$ and a minimum value for $a\left|v_{0}\right| \sin \theta$, namely

$$
a\left|v_{0}\right| \sin \theta>\frac{\pi \hbar c}{4}
$$

The graphical method for $\xi \in \mathbb{R}$ is illustrated in fig. 2. The solutions for bound states are given by the intersection of the curves represented by $\Sigma$ with the curve represented by $-2 \xi \cot 2 \xi$. Seen as a function of $\xi, \Sigma$ is a two-branch decreasing function which begins with $\Sigma \simeq 2 a \sin \theta \sqrt{\left|v_{0}\right|\left(\left|v_{0}\right|+m c^{2}\right)} / \hbar c$ at $\xi=0$ and ends for some $\xi>0$. The branch of solutions with $E<-m c^{2} \cos \theta\left(E>-m c^{2} \cos \theta\right)$ is more favoured for $\theta<\pi / 2(\theta>\pi / 2)$. Above of critical values of $\left|v_{0}\right|, a$ and $\sin \theta$ there will be a finite sequence of bound states with

$$
\left(n-\frac{1}{2}\right) \frac{\pi}{2}<\xi_{n}<n \frac{\pi}{2}, \quad n=1,2,3, \ldots<\frac{2|v|}{\pi} .
$$

Hence,

$$
\left[\left(n-\frac{1}{2}\right) \frac{\pi \hbar c}{2 a}\right]^{2}<E^{2}-m^{2} c^{4}<\left(n \frac{\pi \hbar c}{2 a}\right)^{2}
$$

with

$$
a\left|v_{0}\right| \sin \theta>\left(n-\frac{1}{2}\right) \frac{\pi \hbar c}{2}
$$

and $\left|v_{0}\right|>m c^{2}$. In point of fact, from (45) one can see that (42) is the condition that there is at least one bound-state solution and that the number of possible bound states depends on the size of $a\left|v_{0}\right| \sin \theta$. The number of possible bound states is determined by the maximum value of $n$ satisfying inequality (45). Numerical solutions for the eigenenergies corresponding to $n=1$ as a function of $v_{0} / m c^{2}$ are shown in fig. 3 for different values of $a$ and $\theta$. The case of a massless fermion, as already discussed before with fulcrum on the charge-conjugation and the chiral-conjugation operations, presents a spectrum symmetrical about $E=0$ and seen as a function of $\theta$ exhibits an additional symmetry about $\theta=\pi / 2$. 


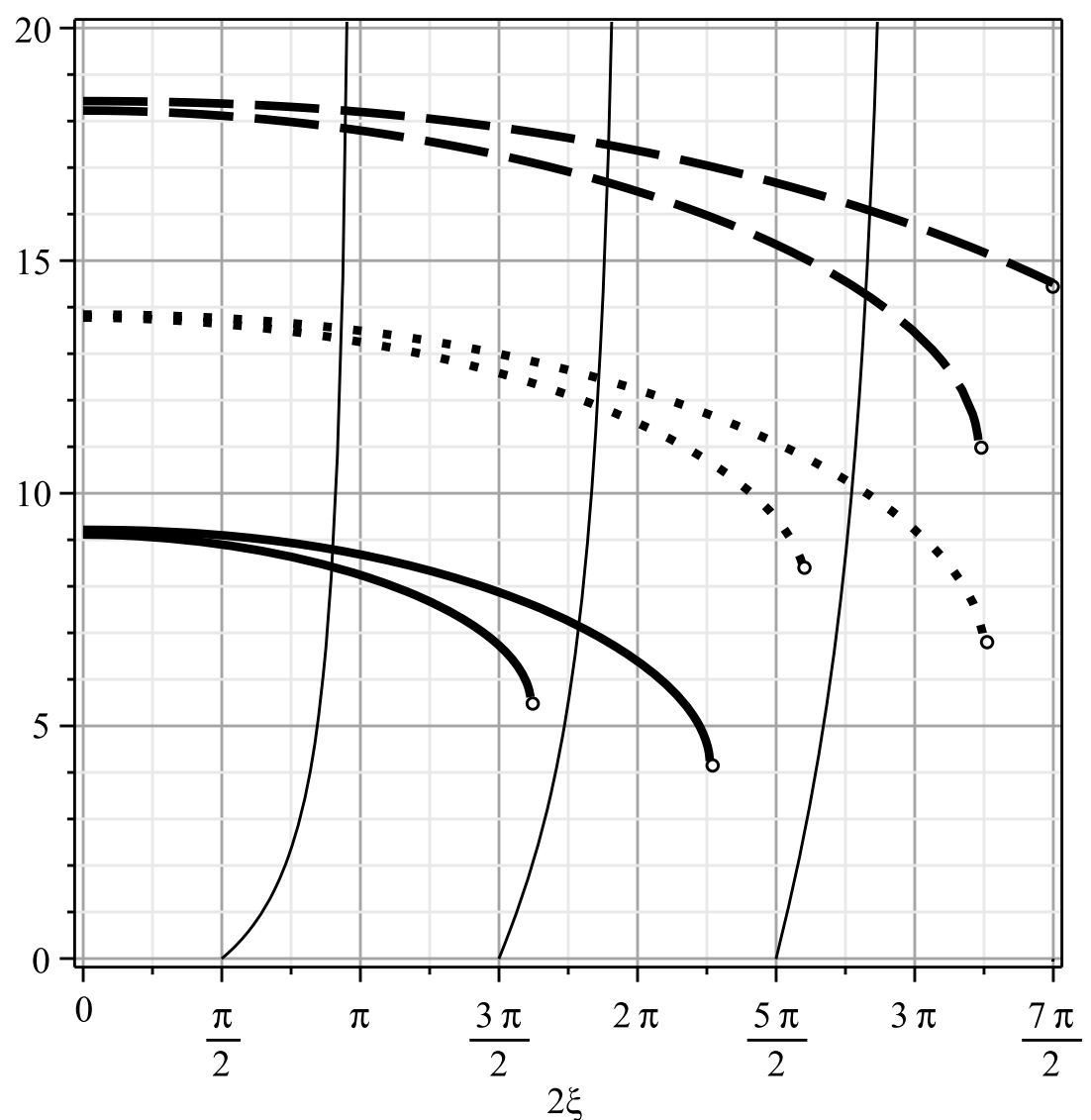

Fig. 2. Graphical solution of $\left|\zeta_{-}\right|+\left|\zeta_{+}\right|=-2 \xi \cot 2 \xi$. The continuous tenuous line for $-2 \xi \cot 2 \xi$. The continuous, dotted and dashed lines for $\left|\zeta_{-}\right|+\left|\zeta_{+}\right|$with $\theta=3 \pi / 8$, and $\left(v_{0}, a / \lambda_{C}\right)$ are equal to $(10,1 / 2),(15,1 / 2)$ and $(10,1)$, respectively. The higher (lower) curve of each pair $\left(v_{0}, a / \lambda_{C}\right)$ corresponds to $E<0(E>0)$.

Exploiting (39) and using the relative amplitudes (31) for $E>-m c^{2} \cos \theta$, we can write

$$
\begin{aligned}
& \frac{B_{+}}{A_{-}}=-e^{-\left(\left|\zeta_{-}\right|-\left|\zeta_{+}\right|\right)} \frac{2 \xi}{\left(\left|\zeta_{-}\right|-\left|\zeta_{+}\right|+2 v\right) \sin \xi}, \\
& \frac{C_{ \pm}}{A_{-}}=-e^{-\left|\zeta_{-}\right|} \frac{\eta_{ \pm}}{\left(\left|\zeta_{-}\right|-\left|\zeta_{+}\right|+2 v\right) \sin \xi} .
\end{aligned}
$$

Note that $\eta_{ \pm}=\xi \cos \xi+\left(\left|\zeta_{+}\right|-v\right) \sin \xi \pm i\left[\left(\left|\zeta_{+}\right|-v\right) \cos \xi-\xi \sin \xi\right]$ in such a way that $\eta_{ \pm}^{*}=\eta_{\mp}$ and $C_{ \pm}^{*}=C_{\mp}$. Therefore, as before, the relative amplitudes for $E<-m c^{2} \cos \theta$ can be obtained by taking the complex conjugate of the right-hand side of (31b) and exchanging the signs of the subscripts of the amplitudes. Figure 4 shows the normalized position probability density for a massive fermion for the Sturm-Liouville solution with $n=1, v_{0} / m c^{2}=5, a / \lambda_{C}=1$ and $\theta=3 \pi / 8$.

\subsection{The case $\mathrm{E}=-\mathrm{mc}^{2} \cos \theta$}

In this case, one finds

$$
v(x)=v_{0}[a+(|x|-a) \Theta(|x|-a)]
$$

As commented before, there is no solution for $\sin \theta=0$. The normalizable solution for $\sin \theta \neq 0$ requires $\left|v_{0}\right|>m c^{2}$ with $\phi=N_{\gtrless} \tilde{\phi} f$. Here,

$$
f=\exp \left(-\frac{\alpha}{2 a}\left\{r[|x| \Theta(|x|-a)+a \Theta(-|x|+a)]+x \operatorname{sgn}\left(v_{0}\right)\right\}\right)
$$

where

$$
\alpha=\frac{2 a \sin \theta}{\lambda_{C}}, \quad \lambda_{C}=\frac{\hbar}{m c}, \quad r=\frac{\left|v_{0}\right|}{m c^{2}}
$$




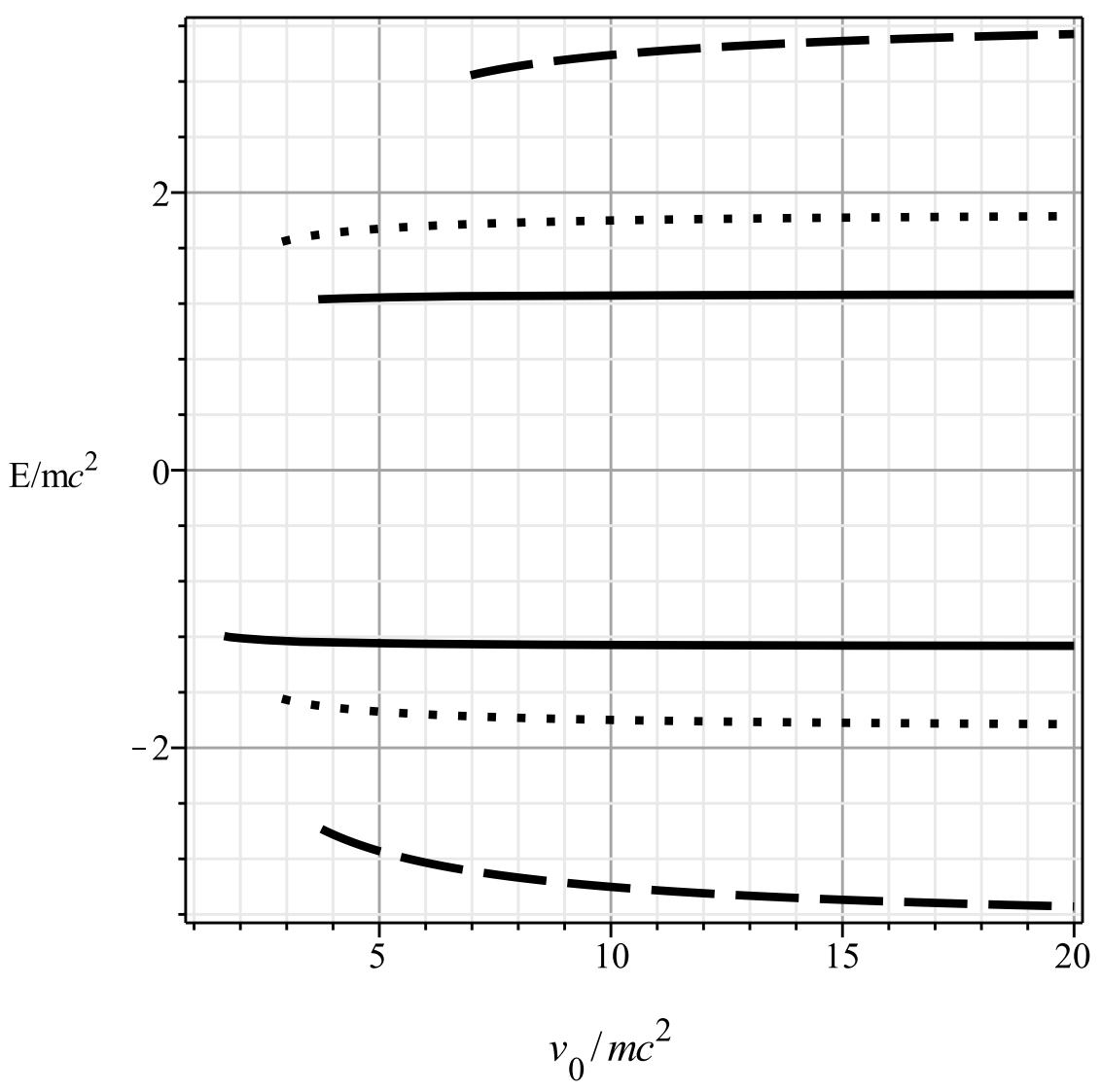

Fig. 3. Energy levels as a function of $v_{0} / m c^{2}$ for $n=1$. The continuous, dashed and dotted lines for $\left(a / \lambda_{C}, \theta\right)$ are equal to $(2,3 \pi / 8),(1 / 2,3 \pi / 8)$ and $(1, \pi / 2)$, respectively.

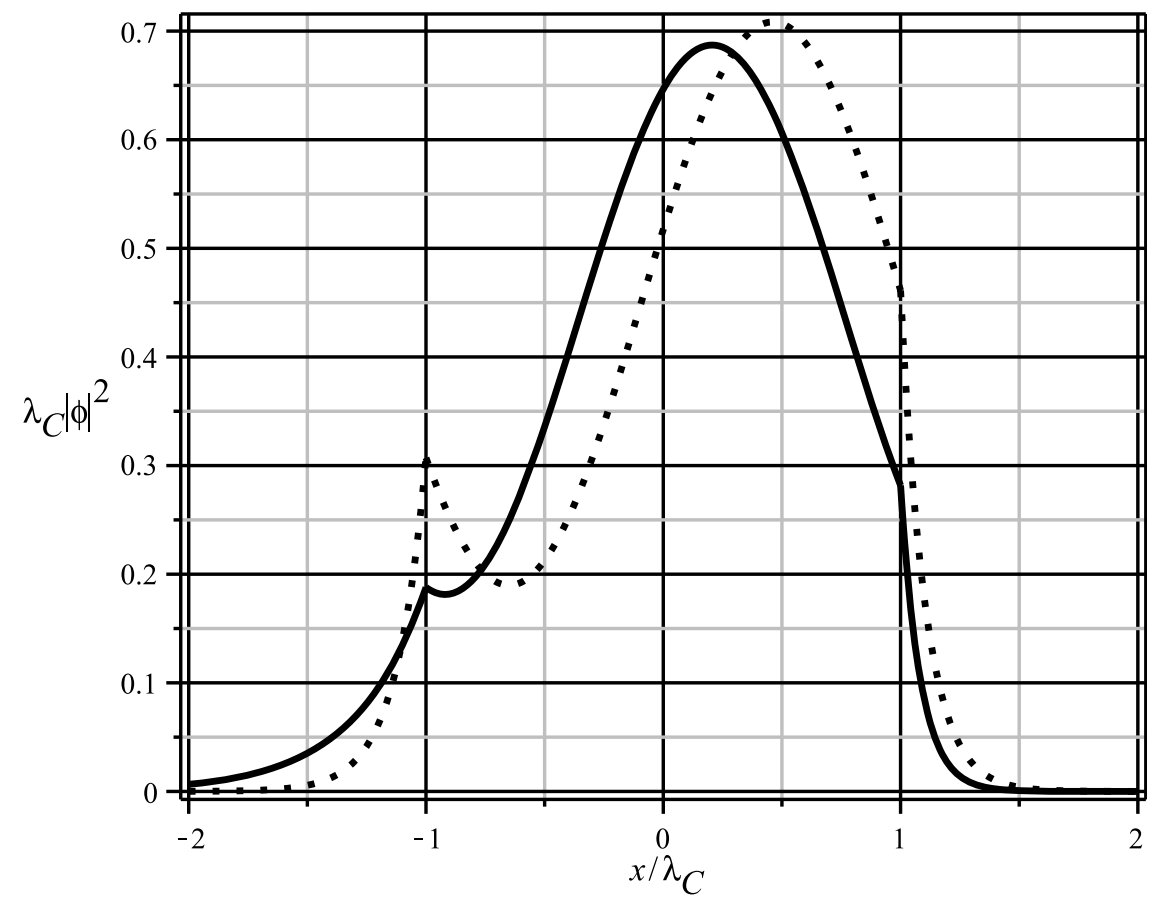

Fig. 4. Position probability density for the Sturm-Liouville solution with $n=1, v_{0} / m c^{2}=5, a / \lambda_{C}=1$ and $\theta=3 \pi / 8$. The continuous line for $E / m c^{2}=+1.7176$, and the dotted line for $E / m c^{2}=-1.7321 . \lambda_{C}=\hbar / m c$ denotes the Compton wavelength of the fermion. 


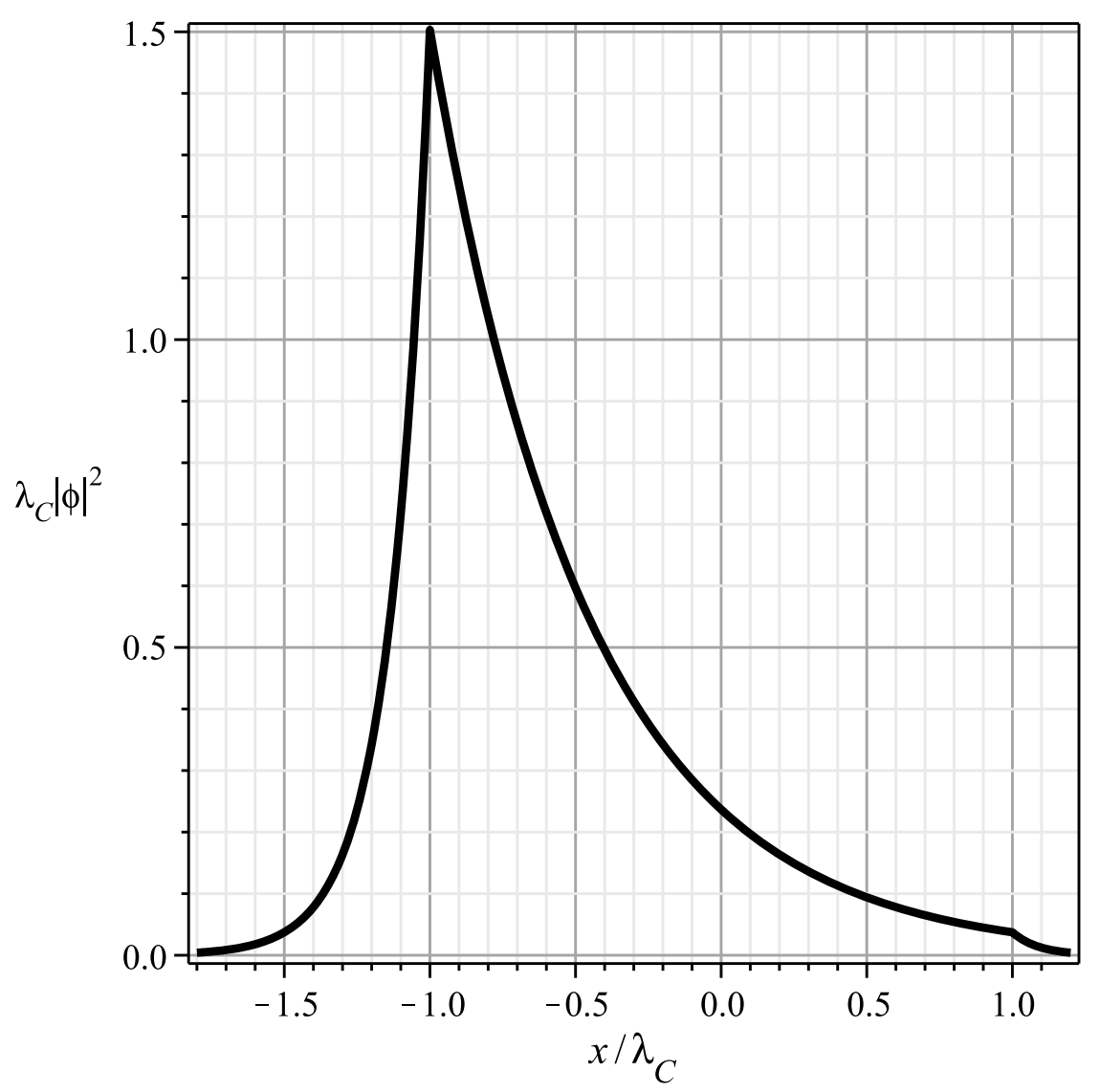

Fig. 5. Position probability density for the isolated solution with $v_{0} / m c^{2}=5, a / \lambda_{C}=1$ and $\theta=3 \pi / 8$.

The normalization condition $\int_{-\infty}^{+\infty} \mathrm{d} x\left(\left|\phi_{+}\right|^{2}+\left|\phi_{-}\right|^{2}\right)=1$ allows one determine $N_{\gtrless}$. One finds the position probability density to be

$$
|\phi|^{2}=\frac{\alpha}{2 a} \frac{r^{2}-1}{r} \frac{e^{r \alpha}}{\cosh \alpha+r \sinh \alpha}|f|^{2} .
$$

Therefore, a massive fermion tends to concentrate at the left (right) region when $v_{0}>0\left(v_{0}<0\right)$, and tends to avoid the origin more and more as $\sin \theta$ decreases. A massless fermion has a position probability density symmetric around the origin (though $r \rightarrow \infty$ and $\alpha \rightarrow 0$ as $m \rightarrow 0, r \alpha \rightarrow$ constant). One can see that the best localization occurs for a pure scalar coupling. In fact, the fermion becomes delocalized as $\sin \theta$ decreases. From

$$
\lim _{a \rightarrow 0} f=\exp \left\{-\frac{\sin \theta}{\lambda_{C}}\left[r+\operatorname{sgn}\left(v_{0} x\right)\right]|x|\right\}
$$

one recovers the value for $\phi$ in the case of the sign potential (at small $a$ ) as in ref. [47]. Figure 5 illustrates the position probability density for a massive fermion with $r=5\left(v_{0}>0\right), a / \lambda_{C}=1$ and $\theta=3 \pi / 8$.

The expectation value of $x$ is given by

$$
\langle x\rangle=-\operatorname{sgn}\left(v_{0}\right) \frac{a}{\left(r^{2}-1\right) \alpha} \frac{c_{3} \cosh \alpha+c_{4} \sinh \alpha}{\cosh \alpha+r \sinh \alpha}
$$

and the fermion is confined within an interval $\Delta x=\sqrt{\left\langle x^{2}\right\rangle-\langle x\rangle^{2}}$ given by

$$
\Delta x=\frac{a}{\sqrt{2}\left(r^{2}-1\right) \alpha} \frac{\sqrt{c_{5} \cosh 2 \alpha+c_{6} \sinh 2 \alpha+c_{7}}}{\cosh \alpha+r \sinh \alpha},
$$


where

$$
\begin{aligned}
& c_{3}=2+r\left(r^{2}-1\right) \alpha, \quad c_{4}=\left(r^{2}-1\right) \alpha-r\left(r^{2}-3\right), \\
& c_{5}=r^{6}+5 r^{2}+2, \quad c_{6}=2 r\left(r^{4}+3\right), \\
& c_{7}=2-r^{2}-r^{6}-2 \alpha^{2}\left(r^{2}-1\right)^{3}-4 r \alpha\left(r^{2}-1\right)^{2} .
\end{aligned}
$$

Again one can see that the fermion becomes delocalized as $\sin \theta$ decreases and that the best localization occurs for a pure scalar coupling. More than this, $|\langle x\rangle| \rightarrow \infty$ and $\Delta x \rightarrow \infty$ as $r \rightarrow 1$. The values for $\langle x\rangle$ and $\Delta x$ as $\alpha \rightarrow 0$ (either in the case of $\sin \theta \rightarrow 0$ or $m \rightarrow 0$ or in the case of the sign potential as in ref. [47]) are given by

$$
\begin{aligned}
& \langle x\rangle \rightarrow-\operatorname{sgn}\left(v_{0}\right) \frac{2 a}{\left(r^{2}-1\right) \alpha}, \\
& \Delta x \rightarrow \frac{2 a \sqrt{r^{2}+1}}{\sqrt{2}\left(r^{2}-1\right) \alpha} .
\end{aligned}
$$

The formula (55b) shows that $\Delta x$ decreases monotonically as $\left|v_{0}\right|$ increases. If $\Delta x$ reduces its extension then $\Delta p$ will must expand, in consonance with the Heisenberg uncertainty principle. Nevertheless, the maximum uncertainty in the momentum is comparable with $m c$ requiring that is impossible to localize a fermion in a region of space less than or comparable with half of its Compton wavelength (see, for example, $[58,59]$ ). This impasse can be broken by resorting to the concepts of effective mass and effective Compton wavelength. Indeed, if one defines an effective mass as $m_{\mathrm{eff}}=m \sqrt{r^{2}+1}$ and an effective Compton wavelength $\lambda_{\mathrm{eff}}=\hbar /\left(m_{\mathrm{eff}} c\right)$, one will find

$$
\Delta x_{\min }=\frac{\lambda_{\text {eff }}}{\sqrt{2} \sin \theta} \frac{r^{2}+1}{r^{2}-1} .
$$

It follows that the high localization of fermions, related to small values of $a$ and strong coupling, never menaces the single-particle interpretation of the Dirac theory even if the fermion is massless $\left(m_{\text {eff }}=\left|v_{0}\right| / c^{2}\right)$. This fact is convincing because the scalar coupling exceeds the vector coupling, and so the conditions for Klein's paradox are never reached. As a matter of fact, (55b) furnishes $(\Delta x)_{\min } \simeq \lambda_{\text {eff }} /(\sqrt{2} \sin \theta)$ for $\left|v_{0}\right| \gg m c^{2}$.

\section{Final remarks}

After reviewing the use of a continuous chiral transformation for solving the Dirac equation in the background of scalar and vector potentials, already applied to the sign potential in ref. [47] and to the smooth step potential in ref. [48], we have extended the methodology to the double-step potential. A common characteristic of all those kink-like potentials is the appearance of an intrinsically relativistic isolated bound-state solution corresponding to the zero-mode solution of the massive Jackiw-Rebbi model in the case of no vector coupling. A finite set of bound-state solutions appears as poles of the transmission amplitude in a strong coupling regime for the double-step potential but all of these solutions coming from the Sturm-Liouville problem tend to disappear as the double-step potential approximates the sign potential. It was also shown that all the bound solutions, including the isolated solution, disappear asymptotically as the magnitude of the scalar and vector coupling becomes the same. Furthermore, we show that due to the sizeable mass gain from the scalar background the high localization of the fermion in an extreme relativistic regime is conformable to comply with the Heisenberg uncertainty principle. Therefore, those bound states can be highly localized by very strong potentials without any chance of spontaneous pair production. A very distinctive feature of the double-step potential is the sequence of transmission resonances not seen in the other kinds of kink potentials (like $\operatorname{sgn} x$ in [47] and tanh $x / \lambda$ in [48]).

The adiabatic method for studying the charge fractionization developed in ref. [42] depends on the background field and it is only reliable for small spacial gradients [60]. The complete set of stationary solutions with sharp discontinuities for the kink-like background field might be useful for additional studies of charge fractionization.

A.S.C. thanks CNPq (Conselho Nacional de Desenvolvimento Científico e Tecnológico) under Project 304743/2015-1 for support.

\section{References}

1. J. Bell, H. Ruegg, Nucl. Phys. B 98, 151 (1975).

2. P.R. Page et al., Phys. Rev. Lett. 86, 204 (2001).

3. J.N. Ginocchio, Phys. Rep. 414, 165 (2005).

4. J.N. Ginocchio, Phys. Rev. Lett. 78, 436 (1997). 
5. J.N. Ginocchio, A. Leviatan, Phys. Lett. B 425, 1 (1998).

6. G.A. Lalazissis et al., Phys. Rev. C 58, R45 (1998).

7. J. Meng et al., Phys. Rev. C 58, R628 (1998).

8. K. Sugawara-Tanabe, A. Arima, Phys. Rev. C 58, R3065 (1998).

9. J.N. Ginocchio, Phys. Rep. 315, 231 (1999).

10. S. Marcos et al., Phys. Rev. C 62, 054309 (2000).

11. P. Alberto et al., Phys. Rev. Lett. 86, 5015 (2001).

12. S. Marcos et al., Phys. Lett. B 513, 306 (2001).

13. J.N. Ginocchio, A. Leviatan, Phys. Rev. Lett. 87, 072502 (2001).

14. P. Alberto et al., Phys. Rev. C 65, 034307 (2002).

15. J.N. Ginocchio, Phys. Rev. C 66, 064312 (2002).

16. C. Ti-Sheng et al., Chin. Phys. Lett. 20, 358 (2003).

17. S.G. Zhou et al., Phys. Rev. Lett. 91, 262501 (2003).

18. G. Mao, Phys. Rev. C 67, 044318 (2003).

19. R. Lisboa et al., Phys. Rev. C 69, 024319 (2004).

20. A. Leviatan, Phys. Rev. Lett. 92, 202501 (2004).

21. J.Y. Guo, Phys. Lett. A 338, 90 (2005).

22. P. Alberto et al., Phys. Rev. C 71, 034313 (2005).

23. J.Y. Guo et al., Phys. Rev. C 72, 054319 (2005).

24. J.Y. Guo et al., Nucl. Phys. A 757, 411 (2005).

25. C. Berkdemir, Nucl. Phys. A 770, 32 (2006).

26. Q. Xu, S.J. Zhu, Nucl. Phys. A 768, 161 (2006).

27. X.T. He et al., Eur. Phys. J. A 28, 265 (2006).

28. R.V. Jolos, V.V. Voronov, Phys. At. Nucl. 70, 812 (2007).

29. S. Chun-Yan et al., Chin. Phys. Lett. 26, 122102 (2009).

30. H. Liang et al., Eur. Phys. J. A 44, 119 (2010).

31. R. Lisboa et al., Phys. Rev. C 81, 064324 (2010).

32. S. Shun-Yan, J.M. Yao, Chin. Phys. C. 34, 1425 (2010).

33. H. Liang et al., Phys. Rev. C 83, 041301(R) (2011).

34. S. Chun-Yan et al., Chin. Phys. Lett. 28, 092101 (2011).

35. B.N. Lu et al., Phys. Rev. Lett. 109, 072501 (2012).

36. A.S. de Castro, P. Alberto, Phys. Rev. A 86, 032122 (2012).

37. P. Alberto et al., Phys. Rev C 87, 031301(R) (2013).

38. P. Alberto et al., J. Phys.: Conf. Ser. 490, 012069 (2014).

39. P. Alberto et al., Phys. Rev. C 75, 047303 (2007).

40. R. Jackiw, C. Rebbi, Phys. Rev. D 13, 3398 (1976).

41. W.P. Su et al., Phys. Rev. Lett. 42, 1698 (1979).

42. J. Goldstone, F. Wilczek, Phys. Rev. Lett. 47, 986 (1981).

43. M.J. Rice, E.J. Mele, Phys. Rev. Lett. 49, 1455 (1982).

44. R. Jackiw, G. Semenoff, Phys. Rev. Lett. 50, 439 (1983).

45. F. Charmchi, S. Gousheh, Phys. Rev. D 89, 025002 (2014).

46. F. Charmchi, S. Gousheh, Nucl. Phys. B 883, 256 (2014).

47. W.M. Castilho, A.S. de Castro, Ann. Phys. (N.Y.) 340, 1 (2014).

48. W.M. Castilho, A.S. de Castro, Ann. Phys. (N.Y.) 346, 164 (2014).

49. W.M. Castilho, A.S. de Castro, J. Phys.: Conf. Ser. 630, 012029 (2015).

50. A.S. de Castro, W.G. Pereira, Phys. Lett. A 308, 131 (2003).

51. L.P. de Oliveira, A.S. de Castro, Can. J. Phys. 90, 481 (2012).

52. L.P. de Oliveira, A.S. de Castro, Int. J. Mod. Phys. E 24, 1550031 (2015).

53. A.S. de Castro et al., Phys. Rev. C 73, 054309 (2006).

54. S. Watanabe, Phys. Rev. 106, 1306 (1957).

55. B.F. Touschek, Nuovo Cimento 5, 754 (1957).

56. A.S. de Castro, Ann. Phys. (N.Y.) 316, 414 (2005).

57. A.S. de Castro, M. Hott, Phys. Lett. A 342, 53 (2005).

58. W. Greiner, Relativistic Quantum Mechanics, Wave Equations (Springer, Berlin, 1990).

59. P. Strange, Relativistic Quantum Mechanics with Applications in Condensed Matter and Atomic Physics (Cambridge University Press, Cambridge, 1998).

60. A.J. Niemi, G. Semenoff, Phys. Rep. 135, 99 (1986). 